\title{
Assessment of variations in arterial tone during different phases of menstrual cycle
}

\author{
E. Umapathy*, S. T. Muthiraparampil, A. Namugowa
}

Department of Human Biology, Faculty of Health Sciences, Walter Sisulu University, NMD Campus, Mthatha, South Africa

Received: 13 February 2019

Accepted: 12 March 2019

\section{*Correspondence:}

Dr. E. Umapathy,

E-mail: eumapathy48@gmail.com

Copyright: () the author(s), publisher and licensee Medip Academy. This is an open-access article distributed under the terms of the Creative Commons Attribution Non-Commercial License, which permits unrestricted non-commercial use, distribution, and reproduction in any medium, provided the original work is properly cited.

\section{ABSTRACT}

Background: Arterial tone parameters in young African women during the different phases of menstrual cycle were assessed in the present study.

Methods: Out of the 30 student volunteers who participated in the study, only 15 completed the study. Anthropometric data using stadiometer, blood pressure using automated oscillometric pressure gauge were measured. Arterial stiffness parameters at the radial and ECG gated carotid and femoral arteries using sphygmocor tonometry was mesured in two consecutive menstrual cycles at early follicular, ovulation and luteal phase. Estrogen and progesterone concentrations were analyzed using Elisa kits in all three phases.

Results: Estrogen level in ovulation phase and progesterone in luteal phase were higher. Peripheral augmentation index in ovulation phase was higher compared to luteal phase. Pulse pressure amplification value at follicular and luteal phases was higher than in ovulation phase. Pulse wave velocity and pulse pressure amplification was negatively correlated to progesterone in follicular phase. The arterial stiffness increased at ovulation and decreased in follicular and luteal phases of menstrual cycle.

Conclusions: No significant correlation between arterial stiffness parameters and ovarian hormones was found.

Keywords: Menstrual cycle, Pulse wave velocity, Peripheral augmentation index, Sphygmocor

\section{INTRODUCTION}

Arterial compliance and PWV (Pulse wave velocity) are markers of arterial elasticity. ${ }^{1} \mathrm{PWV}$ is a direct measure of stiffness, while AIx (Augmentation Index) is an indirect measure. PWV is a biomarker of vascular damage. ${ }^{2}$ The AIx is a measure of the degree to which the peak of a measured pressure wave is over and above the peak of the incident pressure wave due to the addition of the reflected pressure wave. The AIx is dependent on the timing and magnitude of the reflected waveform and is influenced by the compliance and structure of vessels distal to the site of measurement. ${ }^{3}$
Earlier studies, showed no significant variation in the endothelium dependent brachial artery dilation between various phases of menstrual cycle. ${ }^{4}$ Studies on wave reflection indices showed that there was no variation in central and peripheral artery elasticity, blood pressure, carotid intima-media thickness and serum estradiol and progesterone concentrations at 5 points in the menstrual cycle. These studies also indicated that reflected pressure waves do not vary significantly during the menstrual cycle of healthy premenopausal women. ${ }^{5}$ However, recent studies have shown that there is an association between arterial stiffness, thickness and to some novel markers of risk. ${ }^{1}$ 
Peripheral augmentation index (P AIX) is the ratio of central pulse pressure to the peripheral pulse pressure and is a measure of pressure wave reflection with in systemic circulation. P_AIx was significantly high in luteal compared to the ovulatory phase due to fluctuations in hormonal levels during these phases. $^{6}$ A significant reduction in arterial stiffness during mid-cycle was observed, indicating an estrogen dependent reduction in vascular smooth muscle tone. ${ }^{7}$ However, no significant changes in carotid and brachial blood pressures, carotid artery lumen diameter, or intima media thickness was observed throughout the menstrual cycle. ${ }^{8}$ These data provide evidence that the elastic properties of central, but not peripheral, arteries fluctuate significantly with the phases of the menstrual cycle.

Hence, the present study examined the levels and effects of ovarian hormones, estrogen and progesterone, on arterial stiffness during follicular, ovulation and luteal phases of menstrual cycle.

\section{METHODS}

The study design is Cohort prospective study through a menstrual cycle. Thirty premenopausal women of age 1922 years who are not on oral contraception, non-pregnant, non-smoking, non-drinking, non-obese, non-diabetic and non-hypertensive was included in the study. Decision to use this sample size is guided by some of the similar researches where the sample size used ranged from 15 to 32. Only 15 students completed the study due to various constrains.

Ethical approval was obtained from the Post graduate Education, Training, Research and Ethics Unit (053/2016) of Walter Sisulu University, Mthatha, South Africa. The participants were asked to sign a consent form after they were asked to read the participant information sheet and a questionnaire where the inclusion and exclusion criteria were mentioned.

All the women were asked to fast, abstain from caffeine and alcohol intake for 12 hours before the examination. However, they were asked to keep every day regular habits, exercise and nutrition unchanged during the study period. All the measurements was obtained in a quiet, temperature controlled room at $21-23^{\circ} \mathrm{C}$ in the morning starting from $8 \mathrm{am}-10 \mathrm{am}$. The participants were examined in a sitting position after 5-10 minutes of rest. The examination started with brachial blood pressure measurement followed by pulse wave analysis and pulse wave velocity.

After 5 minutes of rest, brachial blood pressure was recorded from the dominant arm using automated digital oscillometric manometer. Central and mean arterial pressure was calculated from the digitized blood pressure curve. Pulse wave reflection was measured using the Sphygmocor pulse wave analysis system. Radial artery waveforms was recorded non-invasively at the wrist of the dominant arm with an applanation tonometer (SPC301, Miller instruments, Houston, Texas, USA) by flattening, but not occluding the radial artery of the dominant arm with gentle pressure9. The aortic augmentation index (AIx) is calculated as the augmentation of aortic systolic pressure by the reflected pulse wave expressed as a percentage of aortic pulse pressure. ${ }^{9}$ Central pressure waves was derived by use of transfer functions. ${ }^{10}$ Multiple recordings was performed in every participants to accomplish optimal quality control criteria.

The Figure 1 depicts the assessment of large artery arterial stiffness. ${ }^{3}$

The anacrotic shoulder represents the inflection pressure (Pi) which associates the commencement of augmentation of the forward pressure wave by reflected pressure. Incisura is associated with the closing of aortic valve and end of systole (Pes).

During the carotid measurement the participant will lie on the bed with their head tilted to the back and to one side. The operator will feel for the position for the strongest pulse and place the tonometer directly on the top of the skin at this point. A pillow placed across the shoulder of the participant will allow the operator to rest their forearm by standing to one side of the participant's head to ensure that the tonometer and wrist remain steady during the measurement.

During the femoral measurement the patient will lie on the bed. The femoral pulse is best felt by pressing directly backward at a point that is midway between the anterior superior iliac spine and the front of the pubic bone, with the thigh flexed at the hip joint, moved away from the midline of the body and rotated away from the body (Atcor Private limited, Sydney, Australia. 2008).

The pulse wave velocity was determined by calculation of the difference in carotid to femoral path length divided by the difference in $\mathrm{R}$ wave to waveform foot times. The difference in carotid to femoral path length was estimated from the supra-sternal notch to the femoral pulse point and from the supra-sternal notch to the carotid pulse point respectively measured in a direct line.

After completing the data capture, report screen was automatically displayed. Before proceeding with the interpretation of the results, tonometry waveform should be clear and smooth and it is important that the foot of the waveform is easily identified. The quality control parameters used in PWA was displayed to the right of the waveforms, the green dot on the waveforms for calculating timing from the wave to the ECG and their similar location in the waveform (Atcor Private Ltd, Sydney, Australia. 2008).

Central arterial stiffness was estimated by measuring carotid-femoral PWV (aPWV) because the common 
carotid and femoral arteries are located superficially and the distance between them spans most of the length of aorta, the arterial segment that is particularly prone to stiffening. The transit time between the two different sites was measured.

After 5 minutes rest, blood sample was collected from all the participants 3 times during a menstrual cycle. First collection wasat 1-4 days of the cycle when estrogen levels are low before selection of the dominant follicle (early follicular phase), second at 10-14 days of the cycle when estrogen output was high (late follicular phase) and third at 20-24 days of the cycle (mid-luteal) when progesterone peaks. These phases are determined on the basis of previous cycle length and the time of menstruation, using the assumption that the luteal phase duration is 14 days. An ovulation kit was provided to each of the participants after training them how to monitor the LH surge in order to identify the specific day of ovulation. During each visit $10 \mathrm{ml}$ fasting serum samples was collected on those specific days by a trained nurse. Each sample may then be assayed to determine the estradiol and progesterone levels using the ELISA kit.

\section{Statistical analysis}

Differences between groups were tested for significance using independent student $t$ test and analysis of variance
(ANOVA) for quantitative variables. Nonparametric Fried man test (Bonferroni test) was performed to assess hormonal level variation. Stepwise linear regression analysis was performed to identify predictor of PMV. Statistical analysis was performed with STATISTIX version 8.1 (Analytical Software, Tallahassee, FL).

\section{RESULTS}

The mean age, height, weight and body mass index of the participants are presented in Table 1.

Table 1: Anthropometric data.

\begin{tabular}{|ll|}
\hline $\begin{array}{l}\text { Anthropometric } \\
\text { measurements }\end{array}$ & $\begin{array}{l}\text { Mean } \pm \text { SD } / \text { Median } \\
(\text { IQR }) ~ N=15\end{array}$ \\
\hline Age (years) & $18(0)$ \\
\hline Height $(\mathrm{cm})$ & $158.5 \pm 6.4$ \\
\hline Weight $(\mathrm{kg})$ & $54(15.5)$ \\
\hline Body mass index $\left(\mathrm{kg} / \mathrm{m}^{2}\right)$ & $22.6(5.9)$ \\
\hline
\end{tabular}

The data on arterial stiffness parameters, namely peripheral augmentation index, pulse pressure amplification percent, aortic augmentation index corrected at 75 beats per minute and pulse wave velocity and estrogen and progesterone levels in various phases of menstrual cycle are shown in Table 2.

Table 2: Arterial stiffness parameters and the hormone levels of estrogen and progesterone in follicular, ovulation and luteal phases of the menstrual cycle.

\begin{tabular}{|llll|}
\hline Parameters of arterial stiffiness & Follicular phase (15) & Ovulation phase (8) & Luteal phase (12) \\
\hline Periheral systolic blood pressure & $116.6 \pm 2.6$ & $107.5 \pm 2.8$ & $113.3 \pm 3.3$ \\
\hline Peripheral diastolic blood pressure & $65 \pm 1.3$ & $65.8 \pm 1.8$ & $62.7 \pm 1.0$ \\
\hline Mean arterial pressure & $79.7 \pm 1.2$ & $79.3 \pm 1.8$ & $77 \pm 1.1$ \\
\hline Peripheral pulse pressure & $48(22)$ & $39(13)$ & $45.5(20.5)$ \\
\hline Peripheral augmentation index & $-56.1 \pm 3$ & $-45.3 \pm 3.8+$ & $-59.4 \pm 4.1$ \\
\hline Aortic systolic blood pressure & $96.3 \pm 1.4$ & $93 \pm 2.1$ & $93.7 \pm 1.9$ \\
\hline Aortic diastolic blood pressure & $65.9 \pm 1.4$ & $66.7 \pm 1.8$ & $63.8 \pm 1.1$ \\
\hline Aortic pulse pressure & $27(10)$ & $25.5(8.5)$ & $28(11)$ \\
\hline Pulse pressure amplification \% & $172(19) \#$ & $158.5(5.6)+$ & $173(11.5)$ \\
\hline Subendocardial viability ratio & $134.2 \pm 5.5$ & $139.6 \pm 5.5$ & $130.1 \pm 4.6$ \\
\hline Aortic augmentation index & $-1.1 \pm 2.8$ & $7.8 \pm 3.9$ & $-2.4 \pm 3.3$ \\
\hline $\begin{array}{l}\text { Aortic augmentation index @ heart rate of } 75 \\
\text { beats per minute }\end{array}$ & $3(15) \#$ & $9(11)+$ & $-1.5(17)$ \\
\hline Pulse wave velocity (m/sec) & $4.6(0.7)$ & $4.6(0.5)$ & $4.9(0.6)$ \\
\hline Progesterone (nmol/L) & $0.43(0.31) *$ & $0.44(0.63)+$ & $4.6(5.1)$ \\
\hline Estrogen (pg/ml) & $58.8 \pm 2.7 \#$ & $71.7 \pm 3.7$ & $66.9 \pm 4.7$ \\
\hline Heart rate & $72(9)$ & $72(15.5)$ & $72.5(12)$ \\
\hline Transfer time & $146(5)$ & $146(13)$ & $147(4.5)$ \\
\hline
\end{tabular}

*p $<0.05$ phase 1 vs phase $3 ;+p<0.05$ phase 2 vs phase $3 \# p<0.05$ phase 1 vs phase 2 .

No significant change was seen in pulse wave velocity in any of the phases, but there was a significant increase in peripheral augmentation index in ovulation phase compared to luteal phase $(\mathrm{p}<0.05)$. Pulse pressure amplification percentages were significantly higher in follicular and luteal compared to ovulation phase $(\mathrm{p}<0.05)$. Both peripheral augmentation index and the aortic augmentation index corrected at heart rate of 75 
beats per minute was significantly high in ovulation phase compared to other phases $(\mathrm{p}<0.05)$. Pulse pressure amplification was low in ovulation phase $(\mathrm{p}<0.05)$. Progesterone was higher in luteal phase while estrogen was higher in ovulation phase $(\mathrm{p}<0.05)$.

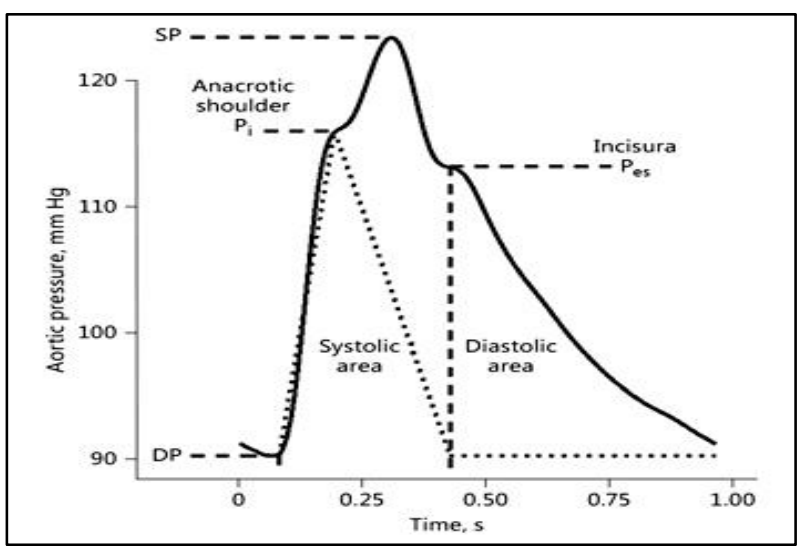

Figure 1: Assessment of large artery stiffness.
Table 3 shows the correlation between progesterone with main arterial stiffness parameters during different phases of menstrual cycle. There was no significant correlation between progesterone and all the arterial stiffness parameters in all the phases of menstrual cycle. The arterial stiffness parameters PWV and PPA were negative at follicular phase meaning there is a negative correlation between the parameters and progesterone in follicular phase. The arterial stiffness parameters P_ALX, A_ALX and A_ALX_75 were negatively correlated to progesterone in ovulation and luteal phases.

Table 4 shows the correlation between estrogen concentrations and the arterial stiffness parameters. There was no significant correlation between estrogen and all the arterial stiffness parameters in all the three phases of menstrual cycle. P_ALX and PPA were negatively correlated to estrogen in follicular and ovulation phases of menstrual cycle except PPA in the ovulation phase. A_ALX and A_ALX_75 are negatively correlated to luteal phase of the menstrual cycle.

Table 3: Correlation of progesterone with main arterial stiffness parameters during different phases of menstrual cycle.

\begin{tabular}{|c|c|c|c|c|c|c|c|}
\hline \multirow{2}{*}{$\begin{array}{l}\text { Arterial } \\
\text { parameters }\end{array}$} & \multirow{2}{*}{ stiffiness } & \multirow{2}{*}{$\begin{array}{l}\mathrm{N}=15 \\
\text { r-value }\end{array}$} & \multirow{2}{*}{$\begin{array}{l}\text { Phase } 1 \\
\text { p-value }\end{array}$} & \multicolumn{2}{|c|}{$\mathbf{N}=8 \quad$ Phase 2} & \multirow{2}{*}{$\begin{array}{l}\mathrm{N}=12 \\
\mathbf{r - v a l u e}\end{array}$} & \multirow{2}{*}{$\begin{array}{l}\text { Phase } 3 \\
\text { p-value }\end{array}$} \\
\hline & & & & r-value & p-value & & \\
\hline PWV & & -0.12 & 0.966 & 0.19 & 0.652 & 0.094 & 0.771 \\
\hline P_ALX & & 0.327 & 0.235 & -0.234 & 0.577 & -0.328 & 0.297 \\
\hline PPA & & -0.435 & 0.105 & 0.165 & 0.696 & 0.360 & 0.250 \\
\hline A_ALX & & 0.336 & 0.221 & -0.229 & 0.585 & -0.344 & 0.274 \\
\hline A_ALX_75 & & 0.223 & 0.425 & -0.141 & 0.739 & -0.396 & 0.203 \\
\hline
\end{tabular}

P_ALx: peripheral augmentation index; PPA: Pulse pressure amplification percent; A_ALx: Aortic augmentation index; PWV: Pulse wave velocity; A_ALx_75: Aortic augmentation index corrected at heart rate of 75 beats per minute.

Table 4: Correlation of estrogen with main arterial stiffness parameters during different phases of menstrual cycle.

\begin{tabular}{|c|c|c|c|c|c|c|}
\hline \multirow{2}{*}{$\begin{array}{l}\text { Arterial stiffiness } \\
\text { parameters }\end{array}$} & \multicolumn{2}{|c|}{$\mathrm{N}=15$ phase 1} & \multicolumn{2}{|c|}{$\mathbf{N}=8$ phase 2} & \multicolumn{2}{|c|}{$\mathrm{N}=12$ phase 3} \\
\hline & r-value & p-value & r-value & p-value & r-value & p-value \\
\hline PWV & .270 & 0.294 & 0.065 & 0.849 & .289 & 0.316 \\
\hline P_ALX & -0.367 & 0.148 & -0.154 & 0.651 & .209 & 0.472 \\
\hline PPA & -0.338 & 0.185 & 0.556 & 0.076 & 0.090 & 0.760 \\
\hline A_ALX & 0.321 & 0.209 & 0.248 & 0.462 & -0.244 & 0.400 \\
\hline A_ALX_75 & 0.054 & 0.838 & -0.544 & 0.084 & -0.302 & 0.295 \\
\hline
\end{tabular}

P_ALx: peripheral augmentation index; PPA: Pulse pressure amplification percent; A_ALx: Aortic augmentation index; PWV: Pulse wave velocity; A_ALx_75: Aortic augmentation index corrected at heart rate of 75 beats per minute.

\section{DISCUSSION}

Pulse wave velocity is a direct measure of stiffness, while Augmentation index is an indirect measure. ${ }^{1}$ The AIx is a measure of the degree to which the peak of a measured pressure wave is over and above the peak of the incident pressure wave due to the addition of the reflected pressure wave. The AIx is dependent on the timing and magnitude of the reflected waveform and is influenced by the compliance and structure of vessels distal to the site of measurement. ${ }^{3}$ Propagation of the pressure pulse from the left ventricle to the systemic circulation is accompanied by reflections from sites of impedance mismatch. These reflections result in backward traveling pressure waves, which augment pressure at the aortic root, this being quantified by the aortic or "central" augmentation index. ${ }^{6}$

The relationship between PWV and AIx remains a matter of debate. ${ }^{1}$ Increased stiffness may precede thickening of 
arteries and this may alter the endothelial function leading to a fall in no synthase activity which may further enhance arterial stiffness which will further lead to increased thickness. ${ }^{11}$ While PWV and aortic AIx seems to undergo changes during aging and body growth, peripheral AIx is not under the influence of aging or body growth. ${ }^{1}$

In the present study, in the ovulation phase, peripheral AIx was decreased while the aortic AIx was increased. However, the pulse pressure amplification percent was significantly increased in follicular and luteal phases compared to the ovulation phase. This is partly in line with previous studies. ${ }^{7}$

Progesterone concentration was significantly high in luteal compared to follicular and ovulation phases of menstrual cycle which is in line with other reports. ${ }^{8,12}$ Estrogen was significantly higher in ovulation compared to follicular phase of the menstrual cycle and this is in line with earlier reports. ${ }^{4,5,7,8}$

The aorta and its major branches may reduce or eliminate the normal elastic gradient between the central and peripheral segments of the arterial tree. This pulsatile stress associated with increased pulse pressure may also induce arterial remodelling. ${ }^{13}$ When correlation was made between arterial stiffness parameters and hormonal concentrations, PWV and PPA were negatively correlated to progesterone at follicular phase meaning that as progesterone increases PWV and PPA decreases and vice versa. Peripheral augmentation index was negatively correlated with progesterone in ovulation and luteal phases (Table 3) meaning when progesterone increases arterial stiffness decreases. Peripheral augmentation index, aortic augmentation index and aortic augmentation index corrected to heart rate of 75 beats per minute were negatively correlated to progesterone in ovulation and luteal phases of menstrual cycle. PAIx may be regarded as the amount by which central pressure is reduced relative to peripheral pressure. ${ }^{6}$ So higher the PAIx during ovulation phase, lower the central pressure which would lead to low arterial stiffness. This showed that phases of menstrual cycle didn't change the arterial stiffness.

The pulse pressure amplification showed a moderately low negative correlation with progesterone in follicular phase, a low positive correlation with ovulation and luteal phases and no correlation between progesterone and arterial stiffness index, PPA. Higher PPA is an indication of less arterial stiffness which might be the result of high estrogen present in follicular and higher progesterone in luteal phases. ${ }^{13,14}$ Lower PPA in ovulation and the resulting rise in arterial stiffness in ovulation could be a result of the inhibition of progesterone against the endothelial dependent vasodilatory actions of estrogen. ${ }^{15}$

The study also identified that arterial stiffness parameters such as aortic augmentation index, aortic pulse pressure, augmentation pressure, heart rate, transit time, aortic systolic blood pressure, peripheral pulse pressure, peripheral systolic blood pressure, peripheral diastolic blood pressure, mean arterial pressure, sub-endocardial viability ratio and pulse wave velocity did not change significantly between phases. This is in line with earlier reports. $^{8}$

Pulse wave velocity did not show significant changes between various phases of menstrual cycle. This is in line with the findings of William et al, who confirmed that central PWV didn't vary significantly over the 4 time points of menstrual cycle. ${ }^{4}$ This is also in agreement with earlier reports which suggested that elastic properties of central, but not peripheral arteries fluctuate significantly with phases of menstrual cycle. ${ }^{8}$ The low arterial stiffness during the menstrual cycle might be due to the balanced agonist-antagonistic vasodilatory effects of estrogen and progesterone in phases 1,2 and $3 .^{16}$

Aortic PWV is a major determinant of the pressure load on the heart both through the compliance and the transmission of the forward and reflected pressure waves. It is related to the intrinsic material stiffness of the aorta. 3 However, PAIx is the ratio of central pulse pressure to the peripheral pulse pressure. PAIx is a measure of pressure wave reflection with in systemic circulation is a direct relationship between amplification and augmentation. Pulse pressure propagation and reflection with in the upper limb results in peripheral amplification of the pressure pulse. ${ }^{6}$ Pulse pressure amplification percent in this study showed significant increase in follicular and luteal phases compared to ovulation phase.

\section{CONCLUSION}

Higher pulse pressure amplification in phase 1 and phase 3 are because of vasodilatory effects of estrogen and progesterone respectively. These findings suggest that the menstrual cycle phase affects central, but not peripheral, arterial elasticity.

\section{ACKNOWLEDGMENTS}

Authors would like to thank Department of Human Biology and the Head of Human Biology for supporting the study by providing necessary equipment and the ELISA kit required for the study.

Funding: Research Directorate, WSU for providing the core funding required for the study

Conflict of interest: None declared

Ethical approval: The study was approved by the Institutional Ethics Committee

\section{REFERENCES}

1. Manco M, Nobili V, Alisi A, Panera N, Handberg A. Arterial stiffness, thickness and association to suitable novel markers of risk at the origin of 
cardiovascular disease in obese children. Int $\mathbf{J}$ med Sci. 2017;14:711-20.

2. Vlachopoulos C, O'Rourke M. Genesis of the normal and abnormal arterial pulse. Curr probl Cardiol. 2000;25:303-67.

3. Butlin M, Qasem A. Large artery stiffness assessment using sphygmacor technology. Pulse (Basel). 2017;4:180-92.

4. Williams MR, Westerman RA, Kingwell BA, Paige J, Blomberry PA, Sudhir K, et al. Variations in endothelial function and arterial compliance during the menstrual cycle. J Clin Endocrinol Metab. 2001;86:5389-95.

5. Pappaioannou TG, Protogerou AD, Stamatelopoulos KS, Vavuranakis M, Stefanadis C. Noninvasive methods and techniques for central blood pressure estimation; procedures, validation, reproducibility and limitations. Curr Pharm Des. 2009;15:245-53.

6. Munir S, Guilche RA, Kamalesh RT, Clapp B, Redwood S, Marber M Chowienczyk P. Peripheral augmentation index defines the relationship between central and peripheral pulse pressure. Hypertension. 2008;51:112-8.

7. Madura M, Sandhya TA. Effect of different phases of menstrual cycle on reflexion index, stiffness index and pulse wave velocity in healthy subjects. J Clin Diagn Res. 2014;8:BC01-4.

8. Hayashi K, Miyachi M, Seno N, Takahashi K, Yamazaki K, Sugawara J, Yokoi T, Onodera S, Mesaki N. Variations in carotid arterial compliance during the menstrual cycle in young women. The Physiological Society. Exp Physiol. 2006;91:465-72.

9. Namugowa A, Iputo J, Wandabwa A, Meeme A, Buga AB. Comparison of arterial stiffness in preeclamptic and normotensive pregnant women from a semi-rural region of South Africa. Clin Exp Hyper. 2017;39:277-83.
10. Karamanoglu M, O'Rourke MF, Avolio AP, Kelly RP. An analysis of the relationship between central aortic and peripheral upper limb pressure wave in man. Eur Heart J. 1993;14:160-7.

11. Zieman SJ, Melenovsky V, Kass DA. Mechanisms, pathophysiology and therapy of arterial stiffness. Arterioscler Thromb Vasc Biol. 2005;25:932-43.

12. Spaczynki RZ, Mitkowska A, Florczak M, Banaszewska B, Krauze T, Wykretowicz A, et al. Decreased large artery stiffness in mid-luteal phase of menstrual cycle in healthy women of reproductive age. Ginekol Pol. 2014;85:771.

13. Laurent S, Boutouyrie P, Asmar R, Gautier I, Laloux B, Guize L. Aortic stiffness is an independent predictor of all-cause and cardiovascular mortality in hypertensive patients. Hypertension. 2001;37:123641.

14. Barbagallo M, Dominguez L, Licata G, Shan J, Bing L, Karpinsk IE, et al. Vascular effects of progesterone: role of cellular calcium regulation. Hypertension. 2001;37:142-7.

15. Minshall RD, Pavnick D, Browne DL, Hermsmeyer K. Nongenomic vasodilator action of progesterone on primate coronary arteries. J Appl Physiol. 2002;92:701-8.

16. Orshal JM, Khalil RA. 'Gender, sex hormones, and vascular tone. Am J Physiol Regal Integr Comp Physiol. 2004;286:233-49.

Cite this article as: Umapathy E, Muthiraparampil ST, Namugowa A. Assessment of variations in arterial tone during different phases of menstrual cycle. Int J Reprod Contracept Obstet Gynecol 2019;8:1810-5. 